\title{
An Optimal Method for Diffusion Parameters of Nonlinear Diffusion Problem of Drug Releasing in 2D-Disc Device by Separate Variable Method
}

\author{
Youyun Li, ${ }^{1,2}$ Jinhui Ouyang, ${ }^{1}$ Jiaohua Qin, ${ }^{3}$ and Yingli Gao ${ }^{2}$ \\ ${ }^{1}$ State Key Laboratory of Simulation and Regulation of Water Cycle in River Basin, Beijing 100038, China \\ ${ }^{2}$ College of Highway Engineering, Changsha University of Science and Technology, Hunan, 410004, China \\ ${ }^{3}$ College of Computer, Central South University of Forestry and Technology, Changsha 410004, China \\ Correspondence should be addressed to Youyun Li; liyouyun8@hotmail.com
}

Received 14 November 2013; Revised 16 February 2014; Accepted 16 February 2014; Published 23 March 2014

Academic Editor: Vassilios C. Loukopoulos

Copyright ( 2014 Youyun Li et al. This is an open access article distributed under the Creative Commons Attribution License, which permits unrestricted use, distribution, and reproduction in any medium, provided the original work is properly cited.

\begin{abstract}
An optimization control model and the corresponding computational method drawing the diffusion parameters of the nonlinear problem for the drug releasing in the 2D-disc device were given in this paper. Firstly, based on the nonlinear diffusion equation of the drug releasing in the $2 \mathrm{D}$-disc device, we used the linear diffusion problem to discrete the nonlinear diffusion problem with the discrete space and the discrete time. Then, by the separate variable method, the solution of the linear problem was given. Next, the least square method based on the separate variable idea (LSMSV) was used to estimate the nonlinear appropriate diffusion parameters. Finally, a numerical example was presented to show that the control model and the numerical method were valid for computing the diffusion coefficient of the nonlinear problem for the drug releasing in the $2 \mathrm{D}$-disc device.
\end{abstract}

\section{Introduction}

In engineering fields, there exist many diffusion processes in many fields such as geomechanics engineering, biomedical science, civil engineering, water pollution, and soil engineering [1-5]. In order to simulate the diffusion processes to obtain their merits, it is important to draw the effective diffusiveness. There are many models for the simulation of the diffusion processes. Most of them are the nonlinear or linear models. For the linear models, most optimal control problems governed by the diffusion equations arose in many scientific and engineering applications such as the water pollution problems and the drug releasing fields [6-10]. There are many various techniques for the identification for the effective diffusiveness based on the linear models. These techniques are based on either empirical or semiempirical models from drug delivery mechanisms or on analytic solutions of the diffusion equation in $2 \mathrm{D}$ or in the special cases [6$8,11,12]$.
However, in the practice application, many diffusion processes are subjected to the nonlinear partial differential equations [6-12]. In order to illustrate the nonlinear diffusion processes in many fields, many nonlinear models are applied to estimate the properties. For many nonlinear diffusion fields, the diffusion coefficients are the functions of the diffusion concentration. Therefore, computing the diffusion parameters is mainly to determinate the parameters of the coefficient function called the diffusion parameters function. The diffusion parameter function of the concentration is considered as the main element to control the diffusion processes. Therefore, many researches were given to determine the parameter function to illustrate the diffusion processes. Many nonlinear optimal models drawing the diffusion parameters depended on both the lab technology and the shape of the container $[9,10,12,13]$. In order to compute the diffusion parameters, many scientists and mathematicians provided some optimal methods to compute the diffusion parameters $[9,10,12]$. Most of them cost a lot of computing time and 
computing memory. Even some of them such as the different method or the finite element method cost more than one week $[9,14]$. Therefore, in order to save the computing time and the computing memory, in our published papers, we had provided some numerical methods based on the separate variable method to compute the diffusion parameters of the linear process in the sphere device. For these reasons, in this paper, we will also propose a new numerical optimal method (the least square method based on the separate variable idea) to extract the diffusion parameters from the nonlinear diffusion problems.

The next two sections will give the nonlinear diffusion problem in the $2 \mathrm{D}$-disc device based on the drug releasing property and the discrete method. Section 3 is devoted to providing the least square method based on the separate variable idea for the optimal control model of the nonlinear diffusion equation system governing the drug releasing process. In Section 4, the numerical example is presented to demonstrate the feasibility and the validity, the convergence of the model, and computing method. Finally, we will discuss the model and computing method and give the future work for the different control model.

\section{The Nonlinear Diffusion \\ Problem in the 2D-Disc Device and the Discrete Linear Problem}

The nonlinear diffusion process in 2D-disc device is governed by the following partial differential equation:

$$
\begin{gathered}
\frac{\partial C(x, y, t)}{\partial t}-\nabla \cdot(D(C) \nabla C)=0, \quad t>0, \quad(x, y) \in \Omega, \\
\frac{\partial C(x, y, t)}{\partial n}=0, \quad t>0, \quad(x, y) \in \partial \Omega, \\
C(x, y, 0)=H(x, y), \quad(x, y) \in \Omega
\end{gathered}
$$

where the drug concentration is uniform in the device and zero in liquid for the initial condition at $t=0$; that is,

$$
H(x, y, 0)= \begin{cases}\frac{M^{0}}{V_{d}}, & (x, y) \in \Omega_{1}, \\ 0, & (x, y) \in \Omega \backslash \Omega_{1}\end{cases}
$$

$M^{0}$ is the total mass of the diffusion material, $V_{d}$ is the volume of $\Omega_{1}$, the diffusion coefficient $D(C)$ is the function of the concentration, $C(x, y, t)$ is the drug releasing concentration, $\Omega_{1}$ is the small disc containing the drug, and $\Omega$ is the big disc containing the liquid shown in Figure 1.

In order to solve the nonlinear diffusion process by the separate variable method, it is necessary to use some linear equations to replace the nonlinear equation (1). The discretion process is given as follows.

Time Discretion. Setting $\Delta t_{i}=t_{i+1}-t_{i}, i=1,2, \ldots, N$, the time $\left[T_{0}, T_{1}\right]$ of the diffusion process is divided into many

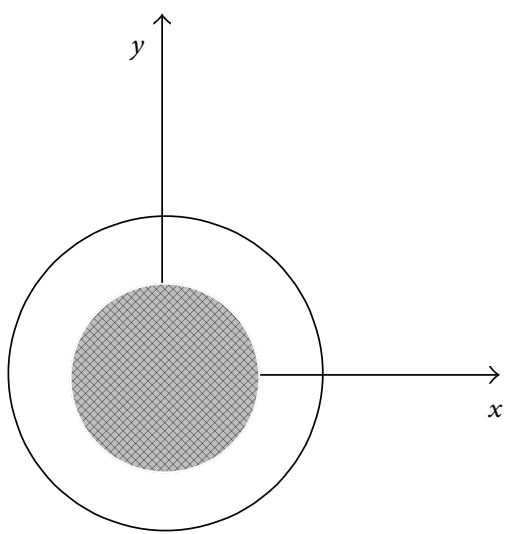

FIGURE 1: Large disc $\Omega$ containing liquid and small disc $\Omega_{1}$ containing drug.

sections $\left[t_{i}, t_{i+1}\right]$ and the concentration $C_{i}$ is computed in $\left[t_{i}, t_{i+1}\right]$.

Space Discretion. Setting $\Delta r_{j}=r_{j+1}-r_{j}, j=1,2, \ldots, K-$ $1, R_{1}=r_{1}<r_{2}<\cdots<r_{k}=R_{2}$, the radius $\left[R_{1}, R_{2}\right]$ is divided into many intervals and the diffusion concentration in the section of $\left[r_{1}, r_{2}\right],\left[r_{2}, r_{3}\right], \ldots,\left[r_{k-1}, r_{k}\right]$ is obtained. So the nonlinear equation system (1) can be changed into $(k-1) * N$ linear equations.

Using the linear diffusion processes to replace the nonlinear diffusion process in the time, it is easy to obtain the following linear equation:

$$
\frac{\partial C_{i}}{\partial t}=D\left(C_{i-1}\right)\left(\frac{\partial^{2} C_{i}}{\partial x^{2}}+\frac{\partial^{2} C_{i}}{\partial y^{2}}\right)
$$

Using the polar coordinate system to replace (3), the above equations can be changed as follows:

$$
\frac{\partial C_{i}}{\partial t}=D\left(C_{i-1}\right)\left(\frac{\partial^{2} C_{i}}{\partial r^{2}}+\frac{1}{r} \frac{\partial C_{i}}{\partial r}\right) .
$$

From the above deduction, the nonlinear diffusion equation of the drug releasing in the section of $\left[t_{i}, t_{i+1}\right]$ and $\left[r_{j}, r_{j+1}\right]$ can be obtained as follows:

$$
\begin{gathered}
\frac{\partial C_{i j}}{\partial t}=D\left(C_{(i-1) j}\right)\left(\frac{\partial^{2} C_{i j}}{\partial r^{2}}+\frac{1}{r} \frac{\partial C_{i j}}{\partial r}\right), \\
i=1,2, \ldots, N, \quad j=1,2, \ldots, K-1 .
\end{gathered}
$$

Because the length of the time and the range of space by the discretion are very small, $D\left(C_{i-1, j}\right)$ can be considered as a constant in $\left[t_{i}, t_{i+1}\right] \times\left[r_{j}, r_{j+1}\right]$. 
In the section of $\left[t_{i}, t_{i+1}\right]$, the linear equations can be represented as follows:

$$
\begin{gathered}
\frac{\partial C_{i j}}{\partial t}=D\left(C_{i-1, j}\right)\left(\frac{\partial^{2} C_{i j}}{\partial r^{2}}+\frac{1}{r} \frac{\partial C_{i j}}{\partial r}\right), \quad t_{i} \leq t \leq t_{i+1}, \\
i=1, \ldots, N, \quad 0<r<r_{j+1}, \quad j=1, \ldots, K-1, \\
\left.\frac{\partial C_{i j}}{\partial r}\right|_{r=r_{j+1}}=0, \\
C_{i j}\left(r, t_{i}\right)= \begin{cases}\frac{M_{i-1, j}}{V_{d_{j}}}, & 0<r<r_{j}, \\
0, & r_{j}<r<r_{j+1},\end{cases}
\end{gathered}
$$

where $V_{d_{j}}=\pi\left(r_{j+1}^{2}-r_{j}^{2}\right)$. Therefore, the concentrations $C_{i j}$ of this equation in $\left[t_{i}, t_{i+1}\right] \times\left[r_{j}, r_{j+1}\right]$ can be obtained and get

$$
\begin{array}{r}
M_{i j}=\int_{0}^{2 \pi} \int_{r_{j}}^{r_{j+1}} C_{i}(r, \theta, t) d r d \theta-\int_{0}^{2 \pi} \int_{r_{j-1}}^{r_{j}} C_{i-1}(r, \theta, t) d r d \theta \\
i=1,2, \ldots, N, \quad j=1,2, \ldots, K .
\end{array}
$$

If $i=0$ and $j=1, C_{01}(r, \theta, 0)=H(r, \theta, 0)$.

To solve $(K-1) \times N$ linear equations, by the separation variable method, we can get the solutions of the nonlinear diffusion equation as follows:

$$
\begin{array}{r}
C_{i j}=\frac{M_{i j}}{V_{d_{j}}}+\sum_{n=1}^{\infty} \frac{2 M_{i j} r_{j}}{V_{d_{j}} r_{j+1} \mu_{n} J_{0}^{2}\left(\mu_{n}\right)} J_{1}\left(\frac{r_{j} \mu_{n}}{r_{j+1}}\right) \\
\quad \times \exp \left(-D\left(C_{(i-1) j}\right)\left(\frac{\mu_{n}}{r_{j+1}}\right)^{2} t_{i}\right) J_{0}\left(\frac{\mu_{n}}{r_{j+1}} r\right), \\
i=1,2, \ldots, N, \quad j=1,2, \ldots, K, \\
C_{i}=\sum_{j=1}^{N} \sum_{l=1}^{i} C_{l j}(r, \theta, t), \quad r \in\left(r_{j}, r_{j+1}\right), i=1,2, \ldots, K .
\end{array}
$$

Equation (9) can be changed into the following formula:

$$
\begin{aligned}
C_{i}=\sum_{j=1}^{N}\left(\sum _ { l = 1 } ^ { i } \left(\frac{M_{l j}}{V_{d}}+\sum_{n=1}^{\infty} \frac{2 M_{l j} r_{j}}{V_{d} r_{j+1} \mu_{n}^{(1)} J_{0}^{2}\left(\mu_{n}^{(1)}\right)} J_{1}\left(\frac{r_{j} \mu_{n}}{r_{j+1}}\right)\right.\right. \\
\quad \times \exp \left(-D\left(C_{(l-1) j}\right)\left(\frac{\mu_{n}}{r_{j+1}}\right)^{2} t_{l}\right) \\
\left.\left.\quad \times J_{0}\left(\frac{\mu_{n}}{r_{j+1}} r\right)\right)\right), \\
\quad r \in\left(r_{2}, r_{K}\right), \quad i=1,2, \ldots, K-1,
\end{aligned}
$$

where $\mu_{n}^{(1)}(n=1,2, \ldots)$ are the positive roots of $J_{1}(x)$ and $\Gamma(m+1)=m !, m$ is the positive integer and

$$
\begin{array}{r}
J_{1}\left(\mu_{n}\right)=\sum_{m=0}^{\infty}(-1)^{m} \frac{1}{\Gamma(m+1) \Gamma(m+2)}\left(\frac{\mu_{n}}{2}\right)^{2 m+1}, \\
J_{0}\left(\frac{\mu_{n}}{R_{2}} r\right)=\sum_{m=0}^{\infty}(-1)^{m} \frac{1}{\Gamma(m+1) \Gamma(m+1)}\left(\frac{\mu_{n} r}{2 R_{2}}\right)^{2 m} .
\end{array}
$$

\section{Control Problem Drawing Nonlinear Diffusion Parameters}

Problem 1. Search the coefficient functions $D\left(a_{1}, \ldots, a_{L}, C\right)$ to satisfy

$$
\begin{gathered}
\min _{D>0}\left\{\left(M_{T 1}-M_{T 1}^{0}\right)^{2}+\left(M_{T 2}-M_{T 2}^{0}\right)^{2}\right. \\
\left.+\cdots+\left(M_{T e}-M_{T e}^{0}\right)^{2}\right\}
\end{gathered}
$$

where $M_{T 1}^{0}, M_{T 2}^{0}, \ldots, M_{T e}^{0}$ are the given experimental data and $M_{T 1}, M_{T 2}, \ldots, M_{T e}$ are the computed data by the following equations:

$$
M_{T i}=\sum_{t_{k} \leq T_{i}, j=2}^{K} M_{k j}
$$

\section{Least Square Method Based on Separate Variable Method for Solving Optimal Control Problem}

Let

$$
\begin{aligned}
E(D)= & \left(M_{T 1}-M_{T 1}^{0}\right)^{2}+\left(M_{T 2}-M_{T 2}^{0}\right)^{2} \\
& +\cdots+\left(M_{T e}-M_{T e}^{0}\right)^{2}=\left(M-M^{*}\right)^{T}\left(M-M^{*}\right),
\end{aligned}
$$

where $M=\left(M_{T 1}\left(a_{1}, \ldots, a_{L}\right), \quad M_{T 2}\left(a_{1}, \ldots, a_{L}\right), \ldots\right.$, $\left.M_{T e}\left(a_{1}, \ldots, a_{L}\right)\right)^{T}$, and $M^{*}=\left(M_{T 1}^{0}, M_{T 2}^{0}, \ldots, M_{T e}^{0}\right)^{T}$.

For an initial diffusion parameter point $D_{1}\left(a_{1}, \ldots, a_{L}\right)$, Problem 1 can be solved iteratively by the following deduction. If $a_{1}^{i}, \ldots, a_{L}^{i}$ are the $i$ th approximation and $\delta a_{1}^{i}, \ldots, \delta a_{L}^{i}$ are the $i$ th increment of $a_{1}, \ldots, a_{L}$, respectively, in each step, an increment $\delta D_{i}\left(\delta a_{1}^{i}, \ldots, \delta a_{L}^{i}\right)$ will be computed as follows. To minimize $E\left(D_{i}+\delta D_{i}\right)$ with $\delta D_{i}\left(\delta a_{1}^{i}, \ldots, \delta a_{L}^{i}\right)$, let $X=$ $\left(x_{1}, \ldots, x_{L}\right)=\left(a_{1}, \ldots, a_{L}\right), f_{T i}(X)=M_{T i}-M_{T i}^{0}, F(X)=$ $E(D)$, so $F(X)=E(X)$ and $\delta D(\delta X)=\delta D_{i}\left(\delta a_{1}^{i}, \ldots, \delta a_{L}^{i}\right)$. If set

$$
\begin{aligned}
f_{i}(X) & =M_{T i}-M_{T i}^{0} \\
& =\int_{0}^{2 \pi} \int_{r_{2}}^{r_{N}} C_{T_{i}}\left(r, \theta, T_{i}\right) d r d \theta-M_{i}^{0} \quad i=1,2, \ldots, e,
\end{aligned}
$$


where

$$
C_{T_{i}}=\sum_{j=2}^{N} \sum_{l=1}^{\max \left\{l \leq T_{i}\right\}} C_{l j}(r, \theta, t), \quad r \in\left(r_{j}, r_{j+1}\right), i=1,2, \ldots, e,
$$

equation (15) can be written in the following form:

$$
F(x)=\sum_{i=1}^{e} f_{i}^{2}(X)
$$

To solve this problem, suppose $X_{(k)}$ is the $k$ th approximation and let the function $f_{i}(X)$ be the Taylor expansion function at $X_{(k)}$; the minimal point $X_{(k+1)}$ and the $(k+1)$ th approximation can be computed by the iterative method. The iterative formula is deduced in detail as follows.

Set

$$
\begin{array}{r}
\phi_{i}(X)=f_{i}\left(X_{(k)}\right)+\nabla f_{i}\left(X_{(k)}\right)^{T}\left(X-X_{(k)}\right) \\
=\nabla f_{i}\left(X_{(k)}\right)^{T} X-\left[\nabla f_{i}\left(X_{(k)}\right)^{T} X_{(k)}-f_{i}\left(X_{(k)}\right)\right], \\
i=1,2, \ldots, e,
\end{array}
$$

$$
\phi(X)=\sum_{i=1}^{e} \phi_{i}^{2}(X)
$$

We use $\phi(X)$ to replace $F(X)$ and compute the minimal point of $\phi(X)$ to estimate the function $F(X)$. The least square problem: $\min \phi(X)$ can be solved as follows. Set

$$
\begin{gathered}
A=\left[\begin{array}{c}
\nabla f_{1}\left(X_{(k)}\right)^{T} \\
\vdots \\
\nabla f_{e}\left(X_{(k)}\right)^{T}
\end{array}\right]=\left[\begin{array}{ccc}
\frac{\partial f_{1}\left(X_{(k)}\right)}{\partial x_{1}} & \cdots & \frac{\partial f_{1}\left(X_{(k)}\right)}{\partial x_{L}} \\
\vdots & & \vdots \\
\frac{\partial f_{e}\left(X_{(k)}\right)}{\partial x_{1}} & \cdots & \frac{\partial f_{e}\left(X_{(k)}\right)}{\partial x_{L}}
\end{array}\right], \\
B=\left[\begin{array}{c}
\nabla f_{1}\left(X_{(k)}\right)^{T} X_{(k)}-f_{1}\left(X_{(k)}\right) \\
\vdots \\
\nabla f_{e}\left(X_{(k)}\right)^{T} X_{(k)}-f_{e}\left(X_{(k)}\right)
\end{array}\right]=A X_{(k)}-f_{(k)}, \\
f_{(k)}=\left[\begin{array}{c}
f_{1}\left(X_{(k)}\right) \\
f_{2}\left(X_{(k)}\right) \\
\vdots \\
f_{e}\left(X_{(k)}\right)
\end{array}\right],
\end{gathered}
$$

$$
\begin{aligned}
& \frac{\partial f_{i}\left(X_{(k)}\right)}{\partial x_{l}} \\
&=\int_{r_{2}}^{r_{N}} \int_{0}^{2 \pi} \sum_{j=1}^{N} \sum_{l=1}^{l \leq T_{i}}\left(\frac{M_{l j}}{V_{d}}+\sum_{n=1}^{\infty} \frac{2 M_{l j} r_{j}}{V_{d} r_{j+1} \mu_{n}^{(1)} J_{0}^{2}\left(\mu_{n}^{(1)}\right)}\right. \\
& \times J_{1}\left(\frac{r_{j} \mu_{n}}{r_{j+1}}\right) \\
& \times \exp \left(-D\left(X_{(k)}, C_{(l-1), j}\right)\right. \\
&\left.\times\left(\frac{\mu_{n}}{r_{j+1}}\right)^{2} t_{l}\right) \\
& \times J_{0}\left(\frac{\mu_{n}}{r_{j+1}} r\right) \frac{\partial D\left(X_{(k),} C_{l-1, j}\right)}{\partial x_{l}} \\
&\left.\times\left(\frac{\mu_{n}}{r_{j+1}}\right)^{2} t_{l}\right) d \theta d r .
\end{aligned}
$$

Equation (19) can be written as

$$
\phi(X)=(A X-B)^{T}(A X-B)=X^{T} A^{T} A X-2 B^{T} A X+B^{T} B .
$$

In order to search the stable point of $\phi(X)$, set

$$
\nabla \phi(X)=2 A^{T} A X-2 A^{T} B=0 .
$$

Taking $A$ and $B$ into the above formula, there is

$$
A^{T} A X=A^{T}\left(A X_{(k)}-f_{(k)}\right) .
$$

Moving the right $A^{\mathrm{T}} A X_{(k)}$ to the left hand in the above equation, the following equation can be obtained:

$$
A^{T} A\left(X-X_{(k)}\right)=-A^{T} f_{(k)} .
$$

Obviously, this is a linear algebraic equation about the function value and the first order partial derivative $f_{i}\left(X_{(k)}\right)$ at the point $X_{(k)}$. If matrix $A$ is the full column rank, $A^{T} A$ is a symmetry positive matrix. Therefore, there exists $\left(A^{T} A\right)^{-1}$. We can get the stable point of $\phi(X)$ by (19)

$$
X_{(k+1)}=X_{(k)}-\left(A^{T} A\right)^{-1} A^{T} f_{(k)} .
$$
$F(X)$.

Set $X_{(k+1)}$ as the $(k+1)$ th approximation of stable point

Algorithm 2 (diffusion parameter of nonlinear process). Step 1. Give the initial point $X_{(1)}=\left(a_{1,(1)}, \ldots, a_{n,(1)}\right)$ and set $t_{1}=0, M_{1}=0, k=1, f_{1}\left(X_{(k)}\right)=0$ and the control error $\varepsilon>0$, go to Step 2 .

Step 2. Compute the drug concentration $M_{i j}\left(X_{(k)}\right)$ and $C_{i j}\left(X_{(k)}\right)$ at $t_{i}$ in $\left[r_{j}, r_{j+1}\right]$ by the formula (7) and (8), obtain the mass in all sections $\left[r_{j}, r_{j+1}\right]$ at the time $t_{i}$, and go to Step 3 . 
TABLE 1: Diffusion qualities at the different times with diffusion parameters (0.0003 and 0.0003).

\begin{tabular}{|c|c|c|c|c|c|}
\hline Time (second) & $M$ & Time (second) & $M$ & Time (second) & $M$ \\
\hline 0 & 0 & 160 & 50.0660 & 300 & 65.0085 \\
\hline 20 & 42.1695 & 180 & 52.2352 & 320 & 67.0014 \\
\hline 40 & 42.2755 & 200 & 54.4820 & 340 & 68.9612 \\
\hline 60 & 42.7675 & 220 & 56.6823 & 360 & 70.8841 \\
\hline 80 & 43.7504 & 240 & 58.8197 & 1000 & 95.6678 \\
\hline 100 & 45.5094 & 260 & 60.9205 & 2000 & 97.1432 \\
\hline 120 & 47.8335 & 280 & 62.9829 & 3000 & 97.1432 \\
\hline
\end{tabular}

TABLE 2: The different errors and optimization value based on initial value (0.0001 and 0.0001$)$.

\begin{tabular}{lcccc}
\hline Iterated number & Total error & $\delta a$ & $\delta b$ & Optimization value $(a, b)$ \\
\hline 1 & $2.9804 \times 10^{4}$ & $-3.0152 \times 10^{-4}$ & $1.1501 \times 10^{-3}$ & $\left(-2.0152 \times 10^{-4}, 1.2502 \times 10^{-3}\right)$ \\
2 & $8.2626 \times 10^{3}$ & $1.4436 \times 10^{-5}$ & $1.7564 \times 10^{-4}$ & $\left(-1.8708 \times 10^{-4}, 1.4258 \times 10^{-3}\right)$ \\
3 & $2.9995 \times 10^{3}$ & $1.3370 \times 10^{-4}$ & $-2.3741 \times 10^{-4}$ & $\left(-5.3387 \times 10^{-4}, 1.1884 \times 10^{-3}\right)$ \\
4 & $9.03742 \times 10^{2}$ & $1.5343 \times 10^{-4}$ & $-3.6483 \times 10^{-4}$ & $\left(1.0004 \times 10^{-4}, 8.2360 \times 10^{-4}\right)$ \\
5 & $2.3343 \times 10^{2}$ & $1.1775 \times 10^{-4}$ & $-3.0877 \times 10^{-4}$ & $\left(2.1779 \times 10^{-4}, 5.1482 \times 10^{-4}\right)$ \\
6 & $4.6960 \times 10$ & $6.0218 \times 10^{-5}$ & $-1.6193 \times 10^{-4}$ & $\left(2.7801 \times 10^{-4}, 3.5290 \times 10^{-4}\right)$ \\
7 & 5.0935 & $1.7408 \times 10^{-5}$ & $-4.3911 \times 10^{-5}$ & $\left(2.9542 \times 10^{-4}, 3.0898 \times 10^{-4}\right)$ \\
8 & 4.0114 & $3.1408 \times 10^{-6}$ & $-6.1503 \times 10^{-6}$ & $\left(2.9856 \times 10^{-4}, 3.0283 \times 10^{-4}\right)$ \\
\hline
\end{tabular}

Step 3. Compute the mass $M_{T i}\left(X_{(K)}\right), i=1,2, \ldots, e$. at the outer container $\Omega \backslash \Omega_{1}$ by the formula (13); go to Step 4 .

Step 4. According to the formula (15), compute $f_{i}\left(X_{(k)}\right)=$ $M_{T i}\left(X_{(k)}\right)-M_{T i}^{0}$; get the vector: $f_{(k)}=\left[\begin{array}{c}f_{1}\left(X_{(k)}\right) \\ f_{2}\left(X_{(k)}\right) \\ \vdots \\ f_{e}\left(\dot{X}_{(k)}\right)\end{array}\right]$. Compute the first order partial derivative $a_{i j}=\partial f_{i}\left(X_{(k)}\right) / \partial x_{j}, i=$ $1,2, \ldots, e, j=1,2, \ldots, L$ by formula $(21)$. Get the matrix $A_{k}=\left(a_{i j}\right)_{e \times L}$ by the formula (20); go to Step 5 .

Step 5. Compute $X_{(k+1)}=X_{(k)}-\left(A^{T} A_{k}\right)^{-1} A^{T} f_{(k)}$; go to Step 6.

Step 6. If $\left\|X_{(k+1)}-X_{(k)}\right\|_{L^{2}} \leq \varepsilon$, set $X^{*}=X_{(k+1)}$ and get the optimum coefficient $\left(a_{1}, \ldots, a_{L}\right)=X^{*}$, go to Step 7; otherwise, let $k=k+1$, go to Step 2 .

Step 7. Output the optimal diffusion $\left(a_{1}, \ldots, a_{L}\right)$ and stop the algorithm.

\section{The Numerical Example}

To investigate the feasibility and the validity of the proposed scheme, a numerical example is given in this section. The cylinder device for the drug releasing is divided into the large and small cylinder structures where the small container includes the drug and the large container is filled with the liquid. The radius of small and large cylinder devices are $0.4800 \mathrm{dm}$ and $2.8399 \mathrm{dm}$, respectively, and there is $100 \mathrm{~g}$ of drug in small disc vessel. When $t=0$, the inner concentration is $138.1553 \mathrm{~g} / \mathrm{dm}^{2}$ and the outer is $0 \mathrm{~g} / \mathrm{dm}^{2}$. After some diffusion process in a period of time, the inner and outer concentration will be equal and be $3.9468 \mathrm{~g} / \mathrm{dm}^{2}$; it is

$$
\begin{gathered}
C(r, 0)= \begin{cases}\frac{M^{0}}{\pi R_{1}^{2}}=138.1553, & 0<r<R_{1}, \\
0, & R_{1}<r<R_{2},\end{cases} \\
C\left(r, T_{e}\right)=3.9468 \quad 0<r<R_{2} .
\end{gathered}
$$

In the numerical example, suppose $D(C)=a C+b$ where $a$ and $b$ are the unknown constants. We firstly suppose the given diffusion parameter $(a, b)$ to be $(0.0003,0.0003)$ then compute the diffusion process to obtain the computed data as the experiment data of the concentration shown in Table 1. Next, the above optimal model and the optimal method to estimate the diffusion parameter based on the optimal control model with the separation variable method of the drug releasing in the $2 \mathrm{D}$-disc device are used to illustrate the feasibility and the validity of the LMSV for the nonlinear diffusion process. The following formula is given to compute the total error in order to estimate the convergence rate:

$$
\begin{aligned}
& \text { total error }(k)=\sum_{j=1}^{e}\left(M_{T_{j}}-M_{T_{j} k}^{0}\right)^{2}, \\
& \delta a=a_{k}-a_{k-1}, \quad \delta b=b_{k}-b_{k-1} .
\end{aligned}
$$

Supposing the space interval to be $0.012 \mathrm{dm}$ and the time interval 20 seconds; getting 25 terms in the Bessel function and by the least square method algorithm, the optimal points and their error values in each optimal step are given in the Table 2. In order to illustrate the convergence of the least square algorithm, the optimal increment $\delta a, \delta b$ and the optimal values $(a, b)$ of the diffusion parameter are depicted in Figures 2, 3 and 4. 


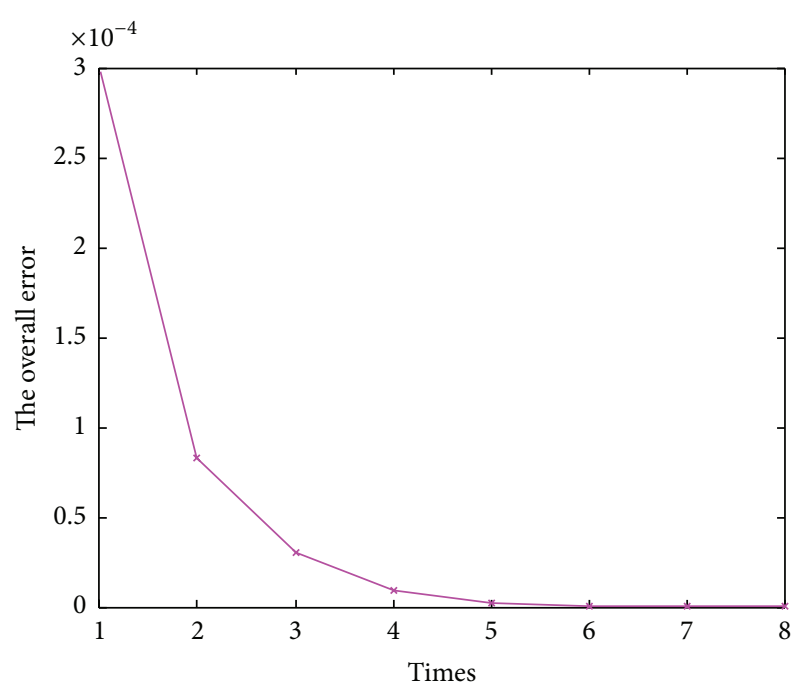

Figure 2: The overall error with iterative times on the initial value for $(0.0001$ and 0.0001$)$.

In order to illustrate the convergence of the algorithm for diffusion parameter of nonlinear process by the least square method by separate variables (LSMSV), we discuss the convergence data as follows: from the second column in Table 2 and Figure 3, the increments $\delta a$ converges very fast because their values become from $-3.0152 \times 10^{-4}$ to $3.1408 \times 10^{-6}$ and from Table 2 and Figure $4 \delta b$ varies from $1.1501 \times 10^{-3}$ to $-6.1503 \times 10^{-6}$. From the last column in Table 2 , the optimized values $a, b$ also become very fast from $\left(-2.0152 \times 10^{-4}, 1.2502 \times 10^{-3}\right)$ to $\left(2.9856 \times 10^{-4}, 3.0283 \times\right.$ $10^{-4}$ ) by only eight iterated steps. Therefore, from Table 2 and the error value in Figures 2, 3 and 4, the data show the error and the increment $\delta a, \delta b$ convergent stately. It is easy to illustrate the convergence of the algorithm for diffusion parameter of nonlinear process by least square method by separate variables idea.

In order to test the convergent velocity, we obtain the computing time for the optimal parameters by the algorithm in the numerical examples. The computing time is 1 minute and 56 seconds by the algorithm for diffusion parameter of nonlinear process. In order to test the merits of the algorithm for diffusion parameter of nonlinear process by LSMSV, it is hard for us to use the algorithm in the paper [9] to compute the parameter values because the computed time is very long. In addition, from the computing processes, it is also easy to understand why the computing velocity becomes very high. Because the algorithm in this paper only computes some of the polynomial functions in each iteration to cost only less than 2 minutes, however, the algorithm in [9] needs to solve millions linear algebra equations in each iteration for the nonlinear diffusion process to cost more than one day. Therefore, from the computing convergent time and the computational theory, we can obtain the conclusion that the convergent velocity of algorithm for diffusion parameter of nonlinear process by LSMSV is very fast.

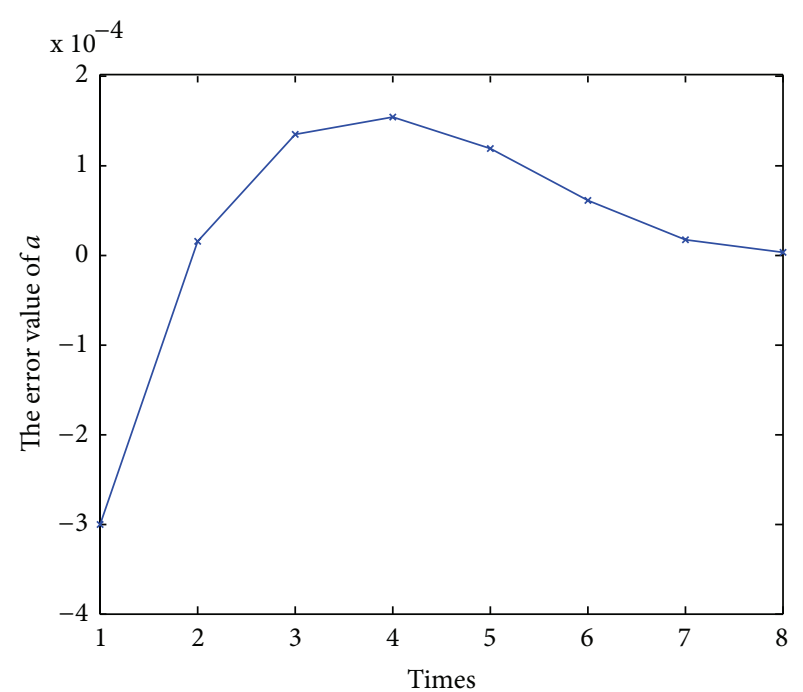

FIgURE 3: The error value of $a$ on the initial value for $(0.0001$ and $0.0001)$.

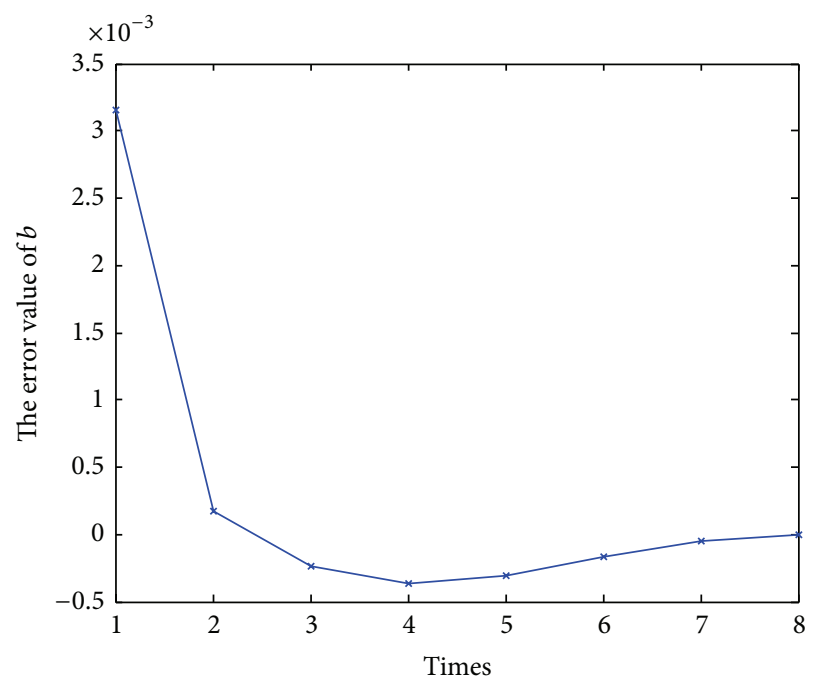

FIgURE 4: The error value of $b$ on the initial value for $(0.0001$ and $0.0001)$.

In order to estimate the validity of the algorithm for diffusion parameter of nonlinear process by LSMSV, the total error values between the experiment values and the computed values in this paper are shown in Figure 5 and the first column in Table 2. The optimal computed drug mass depending on the different optimal diffusion parameters and the experiment mass are depicted in Figure 6. From the first column in Table 2 , the errors of the optimized values $a, b$ and the parameters are only $0.0156 \times 10^{-4}$ and $0.213 \times 10^{-4}$, the error between the optimal computed drug mass $100 \mathrm{~g}$ and the experiment mass data $97.1432 \mathrm{~g}$ is only $2.8568 \mathrm{~g}$, and the relative error of the mass is only $2.9 \%$. The error result shows the algorithm for diffusion parameter of nonlinear process by LSMSV being valid to extract the diffusion parameter of the drug releasing in the disc devices for the nonlinear 


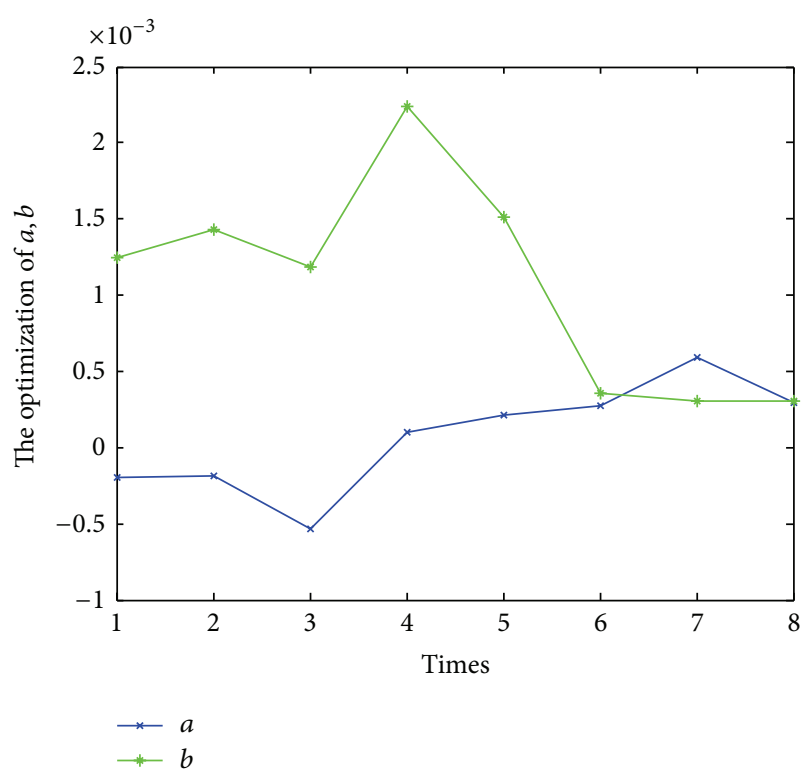

FIGURE 5: The optimization increments of $(a, b)$.

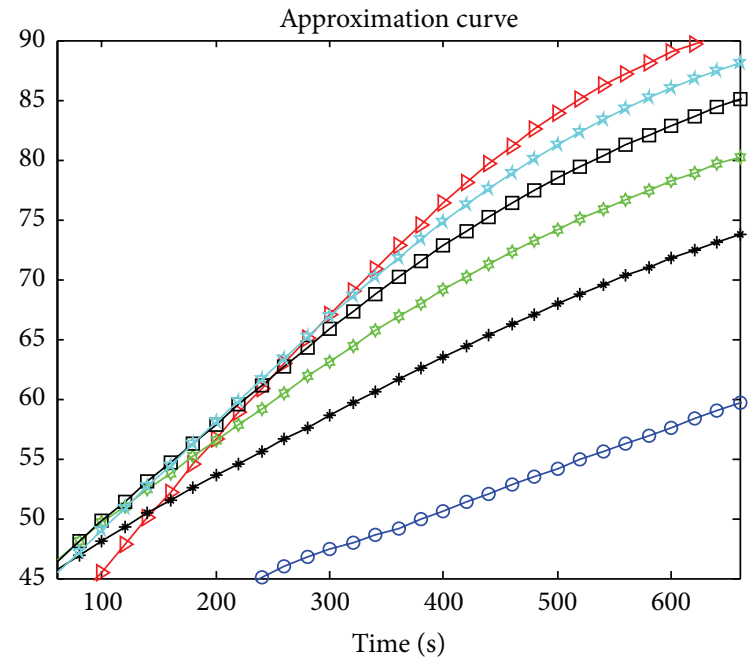

$\triangleright$ Experimental data

$-\odot$ The first optimization value

$\rightarrow$ * The second optimization value

$\rightarrow$ The third optimization value

$\square$ The fourth optimization value

$+\frac{1}{*}$ The fifth optimization value

Figure 6: Computed data in each iteration and experimental data.

diffusion process. From Figures 5 and 6 and the last optimal diffusion parameter value $\left(2.9856 \times 10^{-4}, 3.0283 \times 10^{-4}\right)$, the algorithm is valid to extract the diffusion parameter of the drug releasing for the nonlinear diffusion process in the $2 \mathrm{D}$-disc devices. From the numerical example, comparing the data of all iterative steps of the optimization values and the experimental data, it is easy to get the conclusion that the algorithm is the convergence and effectiveness to extract the diffusion parameters of the nonlinear drug releasing in the 2D-disc devices.

\section{Conclusion}

In this paper, we propose an optimal method to extract the diffusion parameters of the nonlinear diffusion process of the drug releasing in the $2 \mathrm{D}$-disc device based on the separation variable method with discrete time and discrete space. The numerical result given in the previous section demonstrates the feasibility and validity of this algorithm for diffusion parameter of nonlinear process by LSMSV. The effectiveness of this optimal control model to estimate the diffusion parameters for the nonlinear drug releasing in the 2D-disc device is also discussed. How to establish the theorem of the algorithm is our future work.

\section{Conflict of Interests}

The authors declare that there is no conflict of interests regarding the publication of this paper.

\section{Acknowledgments}

This work is supported by National Natural Science Foundation of China (NSFC) Grants (no. 11072041 and 61202496), by State Key Laboratory of Simulation and Regulation of Water Cycle in River Basin (IWHRSKL201205), by State Key Laboratory of Structural Analysis for Industrial Equipment, Dalian University of Technology (GZ1005), by China Postdoctoral Science Foundation (20100480944 and 2012T50692), and by Hunan Provincial Natural Science Foundation of China (13JJ3070, 13JJ2031).

\section{References}

[1] J. Siepmann, A. Ainaoui, J. M. Vergnaud, and R. Bodmeier, "Calculation of the dimensions of drug-polymer devices based on diffusion parameters," Journal of Pharmaceutical Sciences, vol. 87, no. 7, pp. 827-832, 1998.

[2] G. J. Crawford, C. R. Hicks, X. Lou et al., "The Chirila Keratoprosthesis: phase I human clinical trial," Ophthalmology, vol. 109, no. 5, pp. 883-889, 2002.

[3] S. Wang and X. Lou, "Novel mathematicsl models for extracting effective drug diffusivity of porous PHEMA hydrogels: from a planar matrix into a finite external volume," in Proceeding of the ICEAM, Changsha, China, May 2011.

[4] M. Grassi and G. Grassi, "Mathematical modelling and controlled drug delivery: matrix systems," Current Drug Delivery, vol. 2, no. 1, pp. 97-116, 2005.

[5] X. Lou, S. Vijayasekaran, R. Sugiharti, and T. Robertson, "Morphological and topographic effects on calcification tendency of pHEMA hydrogels," Biomaterials, vol. 26, no. 29, pp. 5808-5871, 2005.

[6] M. B. Mellott, K. Searcy, and M. V. Pishko, "Release of protein from highly cross-linked hydrogels of poly(ethylene glycol) diacrylate fabricated by UV polymerization," Biomaterials, vol. 22, no. 9, pp. 929-941, 2001.

[7] J. Zhu and Q. Zeng, "A mathematical theoretical frame for control of air pollution," Science in China. Series D, vol. 32, pp. 864-870, 2002. 
[8] P. E. Price Jr., S. Wang, and I. H. Romdhane, "Extracting effective diffusion parameters from drying experiments," AIChE Journal, vol. 43, no. 8, pp. 1925-1934, 1997.

[9] Y. Li, Z. Xiang, and S. Wang, "Identifying time-dependent drug diffusion parameters in the Cylindrical tube by the finite difference method," in Proceeding of the ICEAM, Changsha, China, May 2011.

[10] D. Parra-Guevara and Y. N. Skiba, "Elements of the mathematical modeling in the control of pollutants emissions," Ecological Modelling, vol. 167, no. 3, pp. 263-275, 2003.

[11] J. C. Fu, C. Hagemeir, and D. L. Moyer, "An unified mathematical model for diffusion from drug polymer composite tablets," Journal of Biomedical Materials Research, vol. 10, no. 5, pp. 743758, 1976.

[12] A. Hukka, "The effective diffusion coefficient and mass transfer coefficient of nordic softwoods as calculated from direct drying experiments," Holzforschung, vol. 53, no. 5, pp. 534-540, 1999.

[13] C. R. Hicks, G. J. Crawford, X. Lou, D. T. Tan et al., "Cornea replacement using a synthetic hydrogel cornea, AlphaCor: device, preliminary outcomes and complications," Eye, vol. 17, pp. 385-392, 2003.

[14] Y. Li, Z. Xiang, X. Xiang, and S. Wang, "A computer algorithm for optimizing to extract effective diffusion coefficients of drug delivery from cylinders," Information Technology Journal, vol. 9, no. 8, pp. 1647-1652, 2010. 


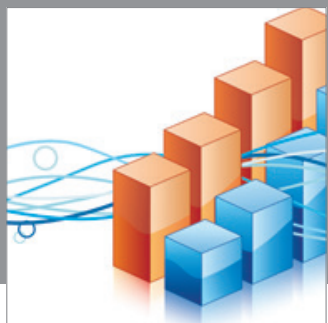

Advances in

Operations Research

mansans

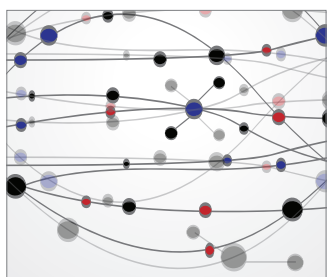

The Scientific World Journal
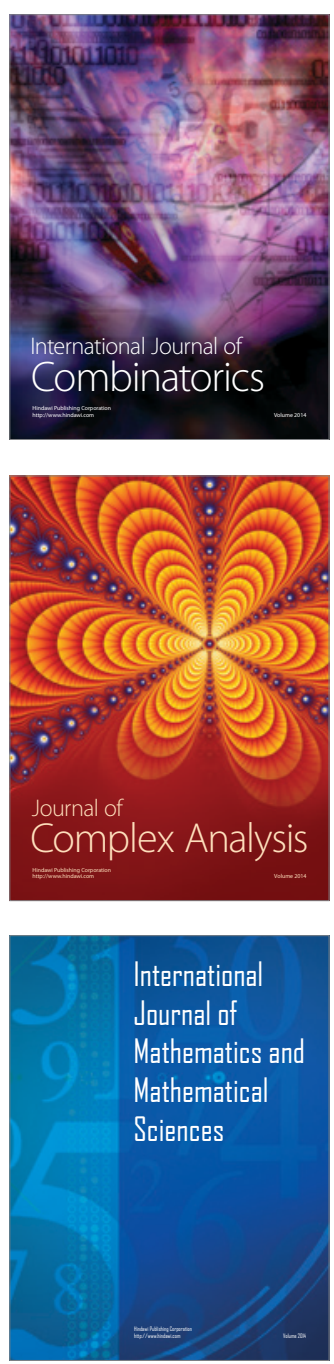
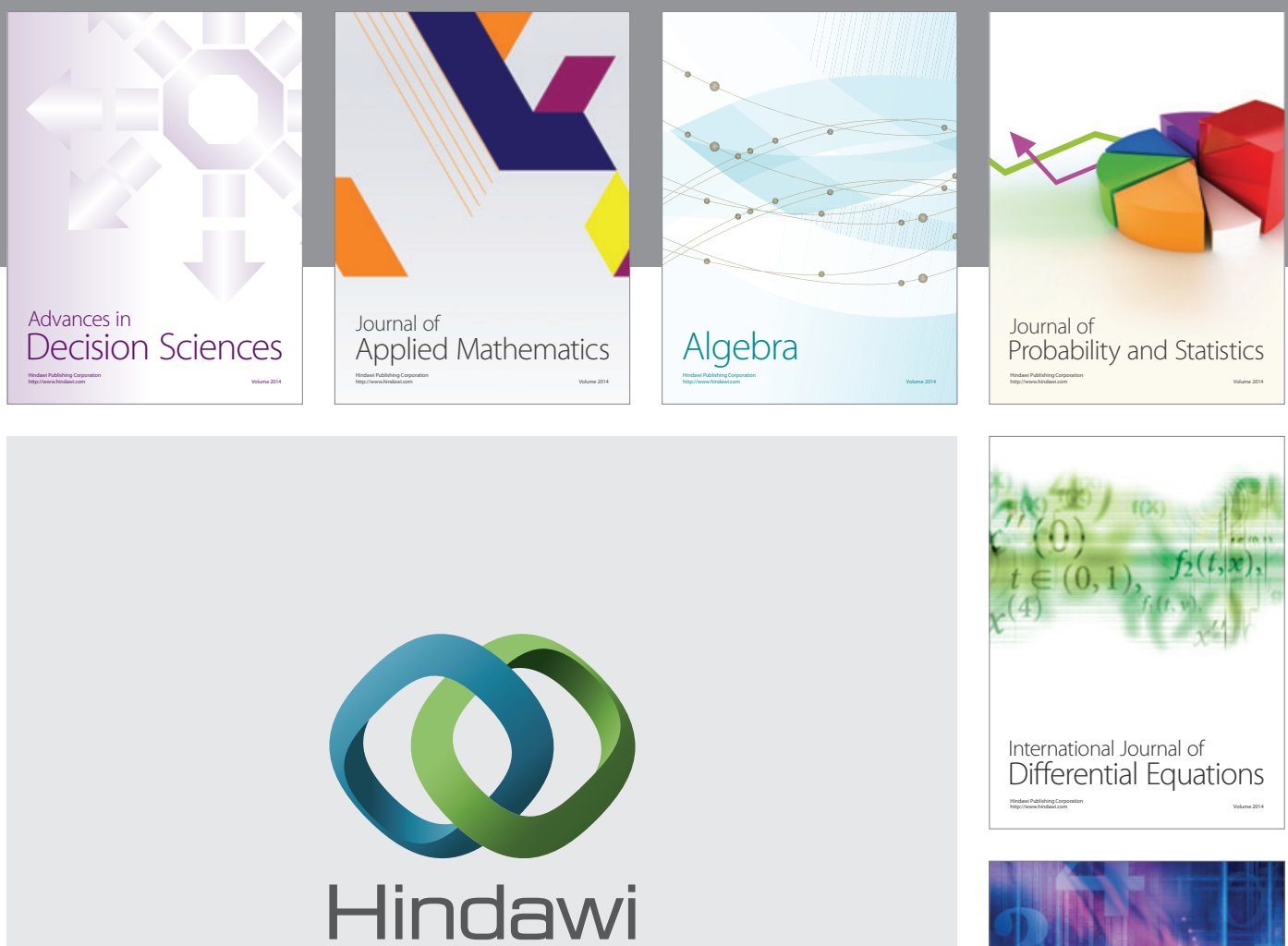

Submit your manuscripts at http://www.hindawi.com
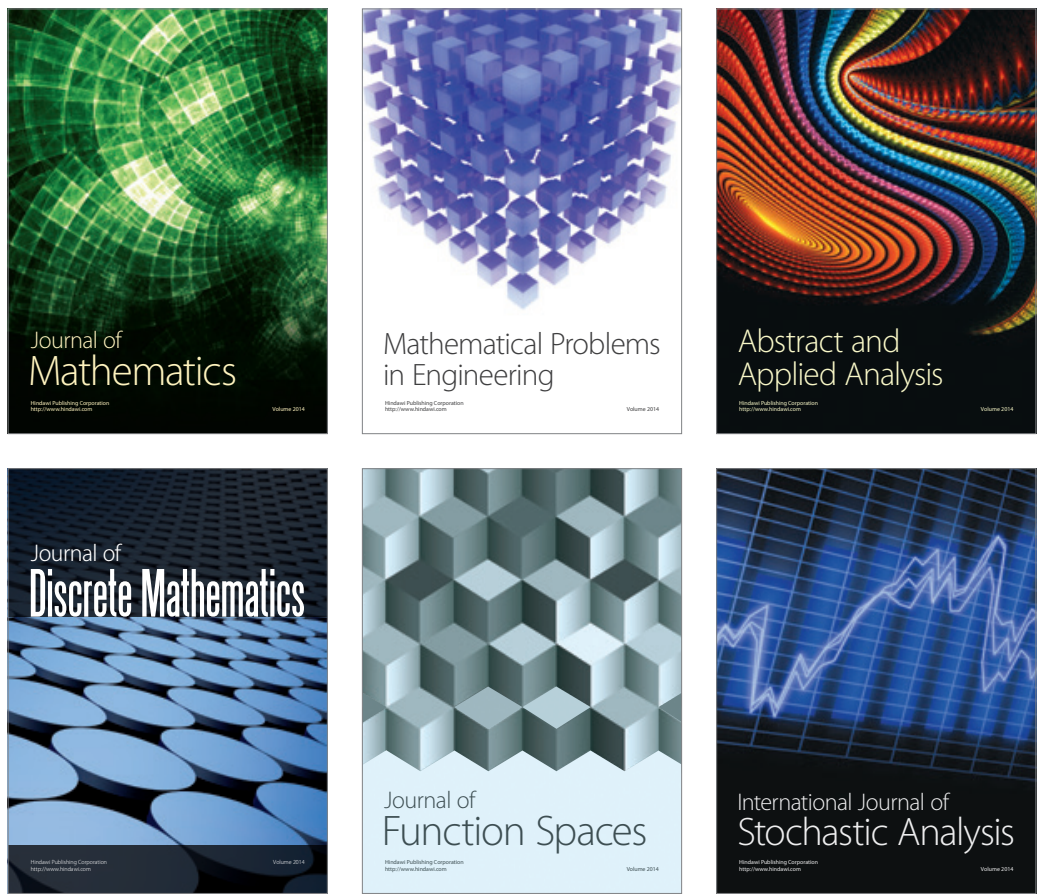

Journal of

Function Spaces

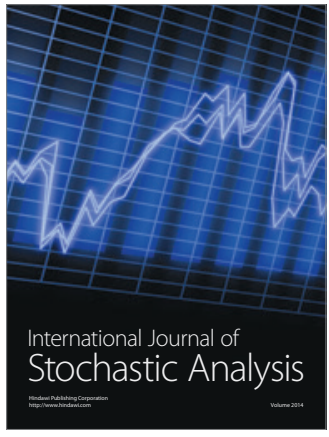

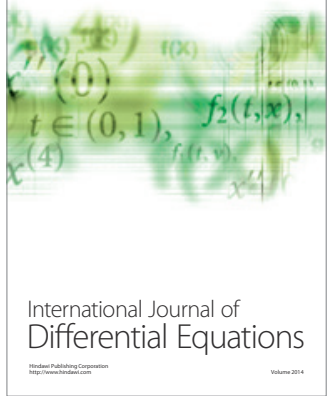
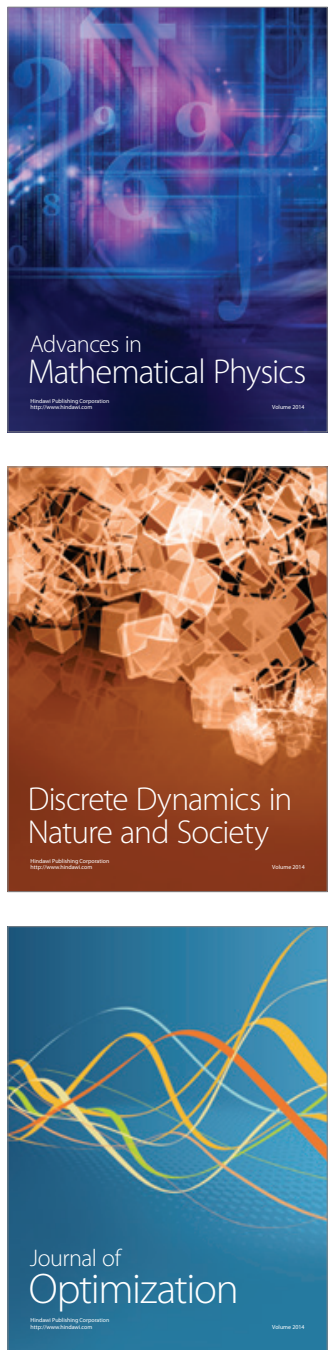